\title{
UNIVERSITYOF
}

FORWARD

THINKING

WESTMINSTER用

WestminsterResearch

http://www.westminster.ac.uk/westminsterresearch

\section{A Fully Adaptive Lattice-based Notch Filter for Mitigation of Interference in GPS}

Arif, S., Coskun, A. and Kale, I.

This is a copy of the author's accepted version of a paper subsequently published in the proceedings of the 15th Conference on PhD Research in Microelectronics and Electronics (PRIME 2019), Lausanne, 15 - 18 Jul 2019, IEEE, doi:10.1109/PRIME.2019.8787822.

The final published version will be available online at:

https://dx.doi.org/10.1109/PRIME.2019.8787822

(C) 2019 IEEE . Personal use of this material is permitted. Permission from IEEE must be obtained for all other uses, in any current or future media, including reprinting/republishing this material for advertising or promotional purposes, creating new collective works, for resale or redistribution to servers or lists, or reuse of any copyrighted component of this work in other works.

The WestminsterResearch online digital archive at the University of Westminster aims to make the research output of the University available to a wider audience. Copyright and Moral Rights remain with the authors and/or copyright owners.

Whilst further distribution of specific materials from within this archive is forbidden, you may freely distribute the URL of WestminsterResearch: ((http://westminsterresearch.wmin.ac.uk/)).

In case of abuse or copyright appearing without permission e-mail repository@westminster.ac.uk 


\title{
A Fully Adaptive Lattice-based Notch Filter for Mitigation of Interference in GPS
}

\author{
Syed Waqas Arif, Adem Coskun and Izzet Kale \\ Applied DSP and VLSI Research Group, \\ School of Computer Science and Engineering \\ University of Westminster \\ 115 New Cavendish Street, W1W 6UW, London, United Kingdom \\ waqas.arif@my.westminster.ac.uk, a.coskun@westminster.ac.uk, kalei@westminster.ac.uk
}

\begin{abstract}
Intentional interference presents a major threat to the operation of the Global Navigation Satellite Systems. Adaptive notch filtering provides an excellent countermeasure and deterrence against narrowband interference. This paper presents a comparative performance analysis of two adaptive notch filtering algorithms for GPS specific applications which are based on Direct form Second Order and Lattice-Based notch filter structures. Performance of each algorithm is evaluated considering the ratio of jamming to noise density against the effective signal to noise ratio at the output of the correlator. A fully adaptive lattice notch filter is proposed, which is able to simultaneously adapt its coefficients to alter the notch frequency along with the bandwidth of the notch filter. The filter demonstrated a superior tracking performance and convergence rate in comparison to an existing algorithm taken from the literature. Moreover, this paper describes the complete GPS modelling platform implemented in Simulink too.

Keywords-Anti-Jamming, Adaptive Notch Filter, GPS receivers, Lattice-Based Notch Filter.
\end{abstract}

\section{INTRODUCTION}

Global Positioning System (GPS) signal utilizes the Code Division Multiple Access (CDMA) technology, where a periodic pseudorandom code is used for spread spectrum modulation on the transmitter, and the same pseudorandom spreading code is used for de-spreading via acquisition module in the receiver. This spreading of the spectrum causes the information energy to be evenly distributed over a wider bandwidth decreasing the power spectral density. As the spread spectrum signal is de-spread back to original bandwidth, the wideband signal is reconstructed into a narrow band signal, and the power spectral density increases. Therefore, CDMA technology itself carries a certain level of interference mitigation capability. The distance between the satellite and the ground-based receiver is approximately $20,500 \mathrm{~km}$ and the transmitting power of the satellite is low compared to the distance it has to travel. One analogy to illustrate this phenomenon is trying to hear a drop of a paper pin in a large classroom. The received GPS signal on the earth surface is extremely weak $\left(1 \times 10^{-16}\right.$ Watts $)$ and submerged $20 \mathrm{~dB}$ below the thermal noise of the receiver. Hence, this makes GPS signal extremely vulnerable to various intentional and unintentional RF interferences. Under such condition, GPS signal cannot be recovered from the noise and this leads a complete disfunctionality.

Interferences on GPS can be intentional and/or unintentional. Intentional interference includes both spoofing and jamming.
Jammer mainly transmits high power jamming signal to jam GPS signal at a receiver front end so that the receiver is unable to acquire and track the GPS signal. Moreover increasing dependency of critical infrastructure on navigation based services necessitates robust and novel methods to mitigate interference in GPS. Different methods can be employed at the receiver end to mitigate interference such as space-time (antenna array), frequency-domain (FFT based) and timedomain (adaptive filter). Both space-time and frequencydomain method increases the hardware complexity and not compatible with mobile devices [1]. Adaptive Notch Filter (ANF) provides an attractive solution to mitigate time-varying Continuous Wave Interference (CWI) due to its simple realization in real-time requiring less hardware complexity. In [2], authors have proposed techniques based on ANF to mitigate CWI with the adaptation of only the notch center frequency parameter for GPS specific applications. Whereas the published work in [3],[4] and [5] shows how pole contraction parameter $\rho$ can be adapted. In [4], $\rho$ converges to 0.76 and 0.88 . Both in [4] and [5], the authors acknowledge the advantages of adapting the pole contraction parameter $\rho$ for performance enhancement. In [3], $\rho$ is adapted but does not indicate to which value it converges. Whereas in [6], $\rho$ is adapted systematically over time but this method cannot be applied to time-varying interference or abrupt changes in the frequency of the CWI. These observations from the literature motivated the development of a novel fully adaptive notch filter which can simultaneously adapts both notch center frequency and notch bandwidth for GPS based application. The performance of the proposed method is compared with [2] in terms of convergence of the notch center frequency parameter, Signal to Noise Ratio (SNR) and acquisition peak metric at the output of thecorrelator.

The rest of the paper is organized as follows; Section II describes the signals and system modelling of GPS L1 generator in Simulink, Section III describes ANF, Section IV describes the proposed method for the adaptation of pole contraction parameter and Section V presents the simulation results.

\section{Signal AND SYSEM MOdEL}

To model GPS L1 signal as realistic as possible, it is important to know the typical values of different parameters for the link budget of the GPS L1 signal. After converting the received GPS signal to Intermediate Frequency (IF), the bandwidth of final IF 
Table.1 Satellite to Receiver Link Budget

\begin{tabular}{|l|l|}
\hline $\begin{array}{l}\text { Transmitted Signal Power via } \\
\text { Satellite }\end{array}$ & $26.8 \mathrm{dBW}$ \\
\hline Free- Space Path Loss (FSPL) & $182.4 \mathrm{~dB}$ \\
\hline Atmosphere Attenuation & $2 \mathrm{~dB}$ \\
\hline Received Signal Power & $-160 \mathrm{dBW}$ \\
\hline Noise Density $\left(N_{o}\right)$ & $K T_{E}=-204 \mathrm{dBW} / \mathrm{Hz}$ \\
\hline Carrier to Noise Density $\left(C / N_{0}\right)$ & $-160-(-204)=44 \mathrm{~dB}-\mathrm{Hz}$ \\
\hline Front-end Bandwidth $(B)$ & $5 \mathrm{MHz}$ \\
\hline
\end{tabular}

filtering range is from $2 \mathrm{MHz}$ to $20 \mathrm{MHz}$ [7]. The noise power in the final IF stage is given by

$$
N=K T_{E} B
$$

Table 1 Shows the typical characteristics and various parameters of the received GPS signals [8], where $K$ is Boltzmann's constant $\left(1.38 \times 10^{-23} \mathrm{~J} / K\right), B$ is the final IF stage bandwidth and $T_{E}$ is the effective noise temperature (290 $\mathrm{K})$. These values result in noise power of $-137 \mathrm{dBW}$ in $5 \mathrm{MHz}$ bandwidth. As the distance of the satellite from the user is typically of $2 \times 10^{7} \mathrm{~m}$, the FSPL can be calculated by

$$
F S P L=\left(\frac{\lambda}{4 \pi R}\right)^{2}
$$

where $\lambda$ is the carrier wavelength $(0.19 \mathrm{~m}$ at $1575.42 \mathrm{MHz})$ and $R$ is the distance between the transmitter and the receiver. Assuming $R=2 \times 10^{7} \mathrm{~m}$, the FSPL is equal to $182 \mathrm{~dB}$.

\section{A. GPS L1 Signal Generation in Simulink}

Figure 1 shows the modelling of the GPS L1 signal in Simulink with Additive White Gaussian Noise (AWGN) $\eta_{0}(t)$, and the transmitted signal without noise and interference as follows

$$
\begin{aligned}
s^{k}(t)= & \sqrt{2 P_{c}}\left(C^{k}(t) \oplus D^{k}(t)\right) \cos \left(2 \pi f_{L 1} t\right) \\
& +\sqrt{2 P_{P L 1}}\left(P^{k}(t) \oplus D^{k}(t)\right) \sin \left(2 \pi f_{L 1} t\right)
\end{aligned}
$$

Where $P_{c}$ represents the signal power level of Coarse/Acquisition (C/A) code $C^{k}(t)$ with a chipping rate of 1.023 $\mathrm{MHz}$ and $P_{P L 1}$ represents the signal power level of $\mathrm{P}(\mathrm{Y})$ codes $P^{k}(t)$ on L1 frequencies. $C^{k}$ and $P^{k}$ are ranging codes of $k^{t h}$ satellite. $D^{k}(t)$ is the navigation data for satellite $k$ with chipping rate of $50 \mathrm{~Hz}$ and $f_{L 1}$ denotes carrier frequency $1575.42 \mathrm{MHz}$ and $\oplus$ represents modulo- 2 addition.

At the receiver front-end, the signal is obtained by downconverting from $f_{L 1}$ frequency to Intermediate Frequency (IF) $f_{I F}$, which can be expressed as follows

$$
\begin{aligned}
& r(t)=s(t)+\eta_{0}(t)+w_{\text {hop }}(t) \\
& =s\left(t-\tau_{o}\right) \cos \left(2 \pi\left(f_{I F}+f_{D}\right) t+\theta\right)+\eta_{0}(t)+w_{\text {hop }}(t)
\end{aligned}
$$

where $s(t)$ is transmitted GPS signal down-converted to IF frequency of $f_{I F}$ and $f_{D}$ represents Doppler frequency relative to the motion of the satellite with respect to the receiver. $\tau_{o}$ represents the delay in GPS signal and $w_{\text {hop }}(t)$ is the hopfrequency CWI interference signal. The can be modelled as in (5) where $A_{w}$ is the amplitude of the interference signal and $f_{h}$

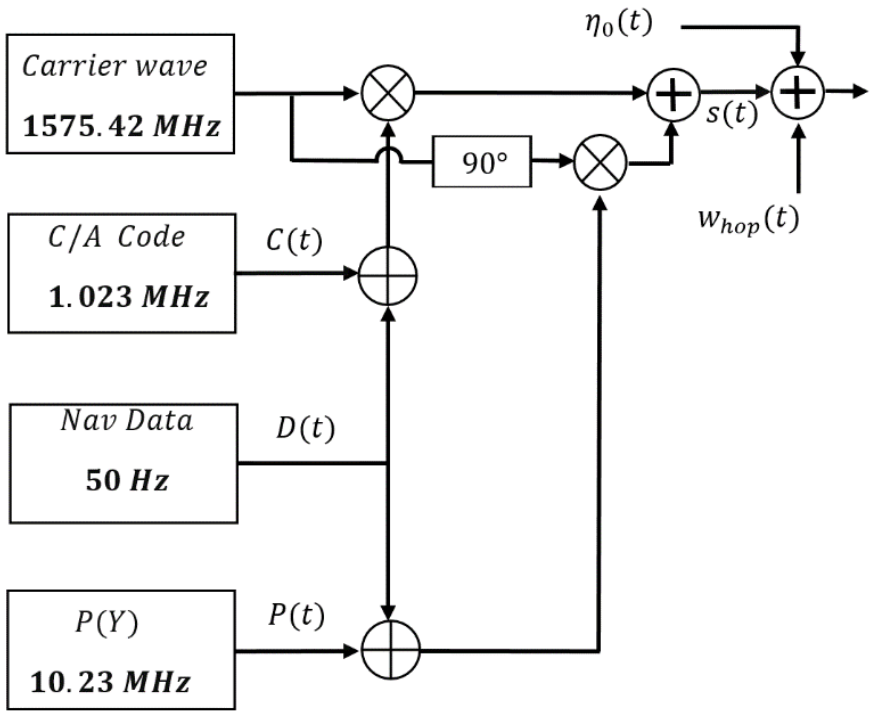

Figure 1. GPS L1 Signal Generation with CWI

is the hop frequency which changes its value at a certain moment in time for a fixed number of samples as shown in Figure 4 with a green dashed line.

$$
w_{\text {hop }}(t)=A_{w} \cos \left(2 \pi f_{h} t+\theta\right)
$$

Jamming-to-Noise Density $\left(J / N_{0}\right)$ for the jamming signal is defined as

$$
J / N_{0}=\frac{A_{w}^{2}}{2 N_{o}}
$$

\section{ADAPTIVE NOTCH FILTERS}

To test different ANF algorithms, GPS L1 signal, modelled in Section II, is used which provides flexibility to control different parameters, such as Doppler shift, code-phase delay, $C / N_{0}$, sampling rate and front-end bandwidth. To remove the CWI added to the signal in (4), Direct form second-order adaptive notch is modelled as given in [2]. The normalized Least Mean Square algorithm for second IIR notch is utilized to update the location of the target frequency. The transfer function of the notch filter is given as follows

$$
H_{n o t c h}(z)=\frac{\left(1-2 \operatorname{Real}\left(z_{0}\right) z^{-1}+\left|z_{0}\right|^{2} z^{-2}\right)}{1-2 k_{\alpha} \operatorname{Real}\left(z_{0}\right) z^{-1}+k_{\alpha}{ }^{2}\left|z_{0}\right|^{2} z^{-2}}
$$

where $k_{\alpha}$ is the notch contraction parameter and $z_{0}$ is the notch location on the unit circle. For the minimization of the cost function $C[n]=E\left\{\left|x_{0}[n]\right|^{2}\right\}$, the derivative of (7) is taken with respect to the parameter $z_{0}$. The expression of the stochastic gradient is given by

$$
\operatorname{grad}(C[n])=-4 x_{0}[n]\left(z_{0}[n-1] x_{e}[n-2]-x_{e}[n-1]\right)
$$

where $x_{0}[n]$ is the output of Auto-Regressive (AR) is part of the filter and $x_{e}[n]$ is the output of Moving-Average (MA) part of the filter and the equation to update the zero of real notch filter becomes

$$
z_{0}[n]=z_{0}[n-1]+\mu_{D} \times x_{0}[n] \operatorname{grad}(C[n])
$$

where $\mu_{D}$ normalized step-size. 


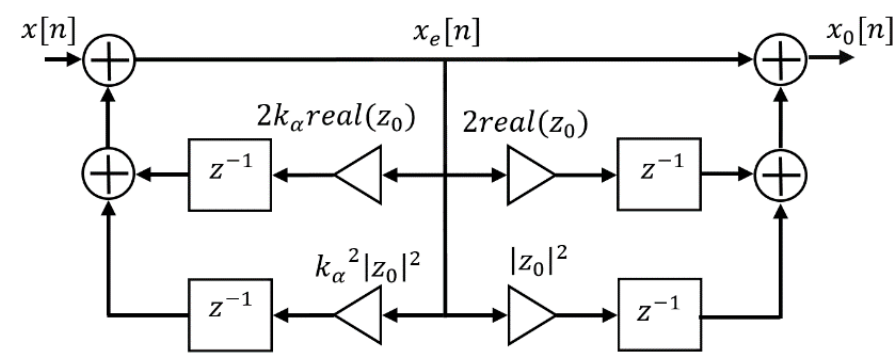

Figure 2. Direct Form $2^{\text {nd }}$ Adaptive Notch Filter

An alternative way to realise a notch filter is an Infinite Impulse Response (IIR) lattice structure. For that purpose, a second structure is employed in the form of an adaptive IIR notch filter with a transfer function (from the input $x_{i n}[n]$ to notch output $y_{L}[n]$ ) expressed in (10), which is depicted in Figure 3. In (10), $\beta$ represents the location of the notch on the unit circle and $\rho$ is pole contraction parameter for lattice notch filter which is equivalent of $k_{\alpha}$ in the direct form IIR notch filter.

$$
H_{L}(z)=\frac{1+\rho}{2} \frac{1-2 \beta(n) z^{-1}+z^{-2}}{1-\beta(n)(1+\rho(n)) z^{-1}+\rho(n) z^{-2}}
$$

The cost function of this filter can be expressed as follows

$$
J(\beta, \rho)=\frac{1}{N} \sum_{n=0}^{N-1} y_{L}^{2}[n]
$$

Hence the gradient signal for adaptation of $\beta$ becomes [3]

$$
\operatorname{grad}\left(J_{\beta}[n]\right)=(1-\rho[n]) x[n-1]
$$

and the update equation for the notch location becomes

$$
\beta[n]=\beta[n-1]-\mu[n] \cdot y_{L}[n] \operatorname{grad}\left(J_{\beta}[n]\right)
$$

where $\mu[n]$ represents time varying step-size [5]. The fixed step size $\mu_{\beta}$ and the instantaneous power estimation of the gradient signal $\phi_{\beta}$ (with a forgetting factor $\gamma$ (usually between $0.9-$ 0.99 ) can adaptively be calculated as follows [3].

$$
\begin{gathered}
\mu[n]=\mu_{\beta} / \phi_{\beta}[n] \\
\phi_{\beta}[n]=\gamma \phi_{\beta}[n-1]+(1-\gamma) \operatorname{grad}\left(J_{\beta}[n]\right)^{2}
\end{gathered}
$$

\section{PROPOSED METHOD}

The adaptive notch filter implementation summarized in Section III doesn't incorporate the adaptation of the pole contraction parameter which is kept constant at a value between 0.80 and 0.90 . In our proposed method to adapt pole contraction parameter, the gradient signal to update $\rho$ is derived by using the definition of the signal at the output of notch filter, which requires the differentiation of (10) with respect to $\rho$ using quotient rule yielding to the result as given in (16). By doing so, a full gradient expression $G_{\rho}(z)$, rather than a partial gradient to update $\rho$ [4][5], is obtained as in (16). Now splitting the denominator and adding term $(1+\rho)$ in the numerator and denominator of the (16), creates the expression as in (17).

$$
\frac{\partial}{\partial \rho}=\frac{0.5\left(1-2 \beta(n) z^{-1}+z^{-2}\right) \cdot\left(1-z^{-2}\right)}{\left(1-\beta(n)(1+\rho(n)) z^{-1}+\rho(n) z^{-2}\right)^{2}}
$$

Based on (17), the update equation for notch bandwidth parameter $\rho$ becomes as shown in (18)

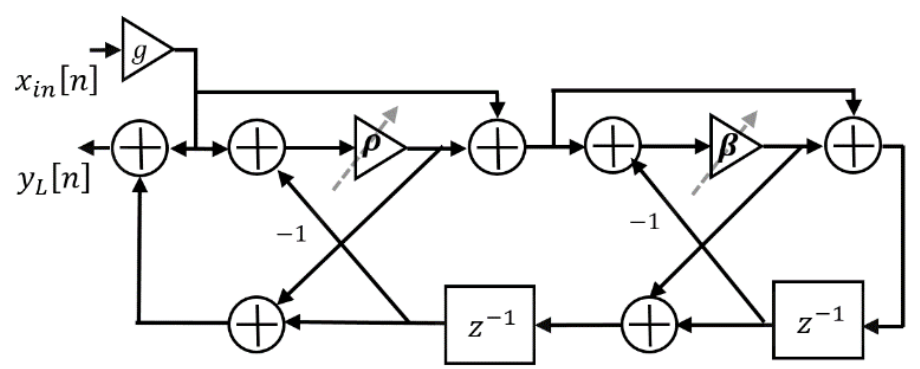

Figure 3. Fully Adaptive Lattice-Based Notch filter

$$
\begin{aligned}
& \frac{\partial}{\partial \rho}\left(H_{L}\right)=\frac{H_{L}(z)}{\frac{1+\rho(n)}{2} \frac{1-2 \beta(n) z^{-1}+z^{-2}}{1-\beta(n)(1+\rho(n)) z^{-1}+\rho(n) z^{-2}}} \times \\
& G_{\rho}(z)\left\{\frac{1-z^{-2}}{1-\beta(n)(1+\rho(n)) z^{-1}+\rho(n) z^{-2}} \times \frac{1}{1+\rho(n)}\right. \\
& \rho[n]=\rho[n-1]+\mu_{\rho} y_{L}[n] g_{\rho}[n] / \phi_{\beta}[n] \\
& \rho[n]<0.95
\end{aligned}
$$

where $y_{L}$ and $\mu_{\rho}$ are the output of the filter and fixed-step size depends on the estimated value of $\phi_{\beta}$. In (14), $g_{\rho}$ is the gradient signal corresponding to $G_{\rho}(z)$. Furthermore, the adaptation of parameter $\rho$ is constrained between 0.70 and 0.95 to produce optimum results in terms of convergence speed and robustness. If the value of $\rho$ is lower than 0.70 the ANF tends to widen notch width which distorts the useful signal and it is observed from this simulation results that by reducing the value of $\rho$ below 0.70 have no significant impact on the convergence speed of the parameter $\beta$. The parameter $\rho$ is reset at the start of new hop-frequency to ensure the fast convergence of $\beta$ to the target frequency. If the value of $\rho$ approaches close to unity (in this case 0.95 ), the previous value of $\rho$ is retained. The parameter $\rho$ is reset when the output of the filter is more than the instantaneous power of the gradient signal which is $\phi_{\beta}$.It is observed from simulation that the signal $\phi_{\beta}$ approaches to zeros whenever ANF have locked on to the new target frequency and once the target frequency is located, it grows in magnitude until next hop-frequency. This effect of the resetting of $\rho$ is demonstrated in the Fig.5. As long as $\phi_{\beta}$ is less than the output of the filter the value of $\rho$ is not updated keeping it at 0.70 just after 1000,2000 and 3000 samples point. Initially $\phi_{\beta}$ is smaller (at the start of each hop frequency) and therefore $\rho$ converges at a faster rate shown in Fig.5. However, $\phi_{\beta}$ get larger in size, $\rho$ is updated at a very slow rate reaching it at the upper limits of 0.95 .

\section{SIMULATIONS RESULTS}

The performance of the lattice based ANF using the proposed fully adaptive notch filter (blue) and the $2^{\text {nd }}$ order direct form ANF from [2] are shown in Fig.4. As can be seen in Fig.4, $\rho$ converges a lot faster than [2] demonstrating a better target frequency detection performance. 


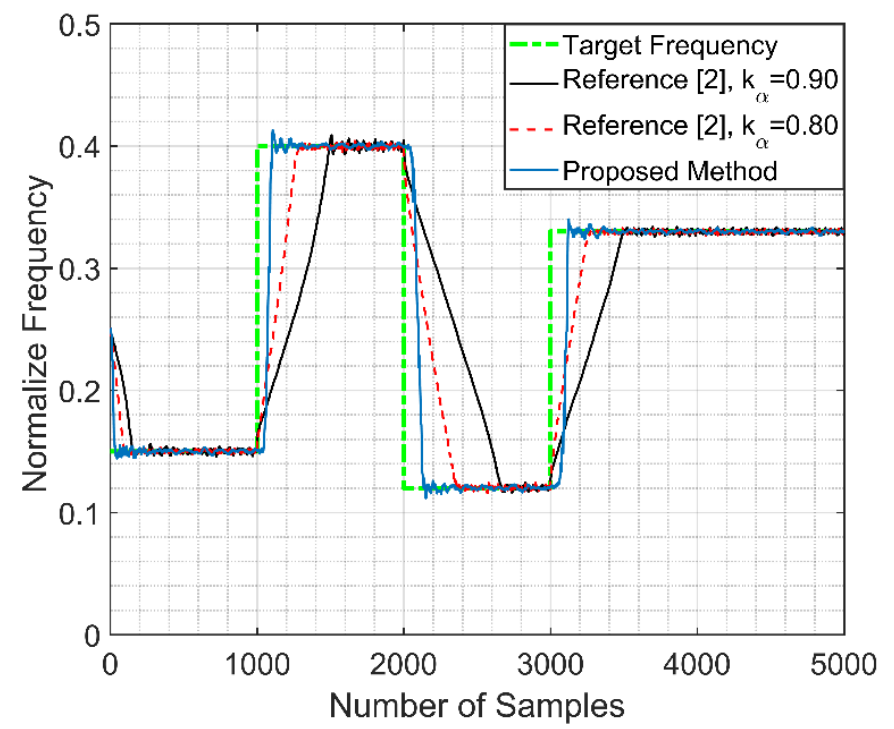

Figure 4. Tracking and Convergence performance of Direct Form IIR Notch filter [2] and proposed method where $\mu_{D}=0.03, \mu_{\beta}=0.02$, $\mu_{\rho}=0.018$ and $\gamma=0.9$.

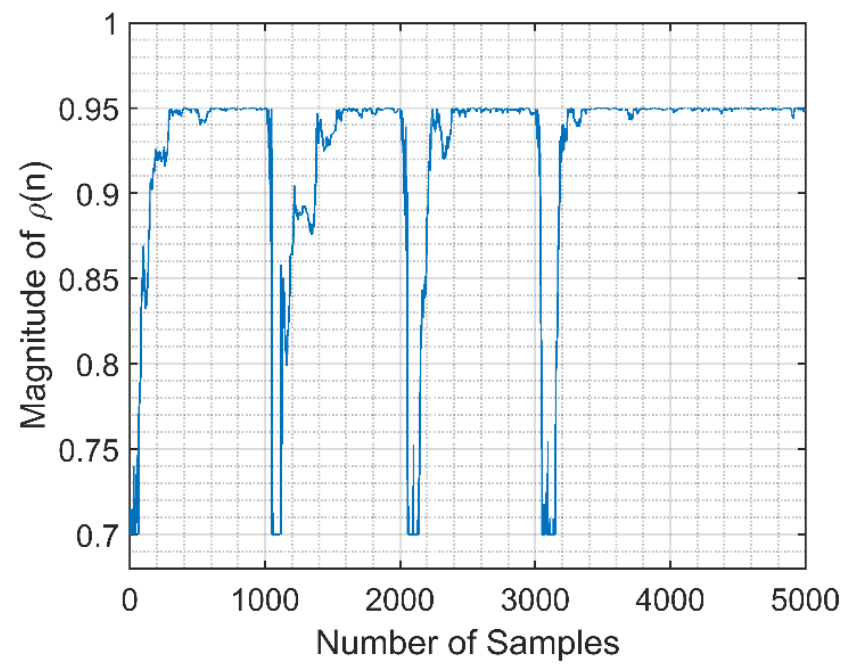

Figure 5. Adaptation of pole contraction parameter $\rho$ using proposed method where $\rho$ resets when $y_{L}[n]>\phi_{\beta}[n]$

Table 2 SNR and $\alpha_{\text {peak }}$ at output of the signal acquisition module with different level input $J / N_{0}(\mathrm{~dB})$

\begin{tabular}{|c|lc|lc|}
\hline$J / N_{0}$ & \multicolumn{2}{|c|}{ Proposed Method } & \multicolumn{2}{c|}{ Reference [2] } \\
\hline$(\mathrm{dB})$ & SNR $(\mathrm{dB})$ & $\alpha_{\text {peak }}$ & SNR(dB) & $\alpha_{\text {peak }}$ \\
\hline 60 & 14.016 & 2.4151 & 13.844 & 2.3044 \\
\hline 100 & 13.9901 & 2.3018 & 12.665 & 1.5880 \\
\hline 120 & $\mathbf{1 3 . 8 6 3 2}$ & $\mathbf{2 . 1 5 5 0}$ & 11.841 & 1.0815 \\
\hline 130 & $\mathbf{1 2 . 9 5 0 1}$ & $\mathbf{1 . 9 9 6 6}$ & 9.4522 & 1.0369 \\
\hline 140 & 9.9161 & 1.1891 & 8.2924 & 1.0687 \\
\hline
\end{tabular}

The performance of both algorithms are evaluated in terms of output SNR at the output of the acquisition module. It is well known that if the SNR is lower 10dB signal is not acquirable [9] GPS system. The second metric is $\alpha_{\text {peak }}$ which is the ratio between the highest and the second highest peaks at the output of the acquisition module and it has to be more than 2 to acquire the GPS L1 signal. Table 2 lists the resulting values of output SNR and $\alpha_{\text {peak }}$ for hop-frequency CWI. Proposed ANF results in higher SNR and $\alpha_{\text {peak }}$ values for different $J / N_{0}$ values. Moreover when the level of $J / N_{0}$ is $120 \mathrm{~dB}$ and $130 \mathrm{~dB}$ the proposed method is able to retain the properties of useful signal hence GPS L1 signal is acquirable but [2] failed to mitigate high level of $J / N_{0}$. Which means by simulataneously adapting both $\rho$ and $\beta$ enhances both mitigating and tracking ability of ANF.

\section{CONCLUSION}

In this research article, two different structures of adaptive notch filter are compared in terms of tracking, convergence speed, and output SNR for GPS applications. A complete GPS transmitter is modelled and presented in Simulink. Simulation results prove that the lattice structure with proposed full gradient algorithm for adaptation of notch bandwidth parameter $\rho$ shows superior performance in term of convergence speed and $S N R$ at the output of the acquisition module. It is demonstrated by simulation results that by simultaneously adapting both $\rho$ and $\beta$, the performance of lattice based adaptive notch filter can be enhanced significantly. This is due to the fact that the transient endure at the start of each subsequent hopfrequency reduces enabling a faster convergence of parameter $\beta$ when parameter $\rho$ set to a lower value of 0.70 . The trade-off between the quality of interference excision and computational complexity of the system is something that is needed to be addressed and further work is being carried out by the authors to simplify the proposed method and develop into a more computationally efficient system.

\section{REFERENCES}

[1] Q. Lv and H. Qin, 'A Novel Algorithm for Adaptive Notch Filter to Detect and Mitigate the CWI for GNSS Receivers', in 2018 Proc. IEEE 3 rd International Conference on Signal and Image Processing (ICSIP), 2018, pp. 444-451

[2] D. Borio, L. Camoriano, and L. L. Presti, 'Two-Pole and Multi-Pole Notch Filters: a Computationally Effective Solution for Interference Detection and Mitigation', in 2007 7th International Conference on ITS Telecommunications, 2007, pp. 1-6.

[3] R. Punchalard and W. Lertvasana, 'Convergence speed improvement for a variable step-size algorithm by using variable notch bandwidth technique', in Proc. International Conference on Neural Networks and Signal Processing, 2003. vol. 1, pp. 852-855

[4] A. Muuma, S. Nishimura, and T. Hinamoto, 'Adaptive IIR notch filter with controlled bandwidth for narrow-band interference suppression in DS CDMA system', in 2003 Proc. of International Symposium on Circuits and Systems, 2003, vol. 4, pp. IV-IV.

[5] A. Mvuma, S. Nishimura, and T. Hinamoto, 'Adaptive optimization of notch bandwidth of an IIR filter used to suppress narrow-band interference', in 2002 Proc. IEEE International Symposium on Circuits and Systems, 2002, vol. 5, pp. V-V.

[6] P. Regalia, Adaptive IIR Filtering in Signal Processing and Control, 1 edition chapter 10. New York: CRC Press, 1994.

[7] E. D. Kaplan and C. Hegarty, Understanding GPS Principles and Applications. Boston: Boston : Artech House, 2006.

[8] M. S. Braasch and A. J. van Dierendonck, "GPS receiver architectures and measurements," Proceedings of the IEEE, vol. 87, no. 1 , pp. 48-64, Jan. 1999

[9] S. W. Arif, A. Coskun, and I. Kale, 'Multi-Stage Complex Notch Filtering for Interference Detection and Mitigation to Improve the Acquisition Performance of GPS', in 2018 14th Conference on Ph.D. Research in Microelectronics and Electronics (PRIME), 2018, pp. 165-168. 